\title{
Odin observations of the Galactic centre in the 118-GHz band ${ }^{\star}$ Upper limit to the $\mathrm{O}_{2}$ abundance
}

\author{
Aa. Sandqvist ${ }^{1}$, B. Larsson ${ }^{1}$, A. Hjalmarson ${ }^{2}$, P. Bergman ${ }^{2}$, P. Bernath ${ }^{3}$, U. Frisk ${ }^{4}$, M. Olberg ${ }^{2}$, \\ L. Pagani ${ }^{5}$, and L. M. Ziurys ${ }^{6}$ \\ 1 Stockholm Observatory, AlbaNova University Center, 10691 Stockholm, Sweden \\ e-mail: aage@astro.su.se \\ 2 Onsala Space Observatory, 43992 Onsala, Sweden \\ 3 Department of Chemistry, University of York, Heslington, York YO10 5DD, UK \\ 4 Swedish Space Corporation, PO Box 4207, 17104 Solna, Sweden \\ 5 LERMA \& UMR8112 du CNRS, Observatoire de Paris, 61 Av. de l'Observatoire, 75014 Paris, France \\ 6 Arizona Radio Observatory, Steward Observatory, University of Arizona, Tucson, AZ 85721, USA
}

Received 22 January 2008 / Accepted 23 February 2008

\section{ABSTRACT}

\begin{abstract}
Aims. The Odin satellite has been used to search for the 118.75-GHz line of molecular oxygen $\left(\mathrm{O}_{2}\right)$ in the Galactic centre. Methods. Odin observations were performed towards the Sgr A* circumnuclear disk (CND), and the Sgr A $+20 \mathrm{~km} \mathrm{~s}^{-1}$ and $+50 \mathrm{~km} \mathrm{~s}^{-1}$ molecular clouds using the position-switching mode. Supplementary ground-based observations were carried out in the 2-mm band using the ARO Kitt Peak 12-m telescope to examine suspected SiC features.

Results. A strong emission line was found at $118.27 \mathrm{GHz}$, attributable to the $J=13-12 \mathrm{HC}_{3} \mathrm{~N}$ line. Upper limits are presented for the $118.75-\mathrm{GHz} \mathrm{O}_{2}\left(1_{1}-1_{0}\right)$ ground transition line and for the $118.11-\mathrm{GHz}^{3} \Pi_{2}, J=3-2$ ground state $\mathrm{SiC}$ line at the Galactic centre. Upper limits are also presented for the $487-\mathrm{GHz} \mathrm{O}_{2}$ line in the $\mathrm{Sgr} \mathrm{A}+50 \mathrm{~km} \mathrm{~s}^{-1}$ cloud and for the $157-\mathrm{GHz}, J=4-3, \mathrm{SiC}$ line in the Sgr A +20 and $+50 \mathrm{~km} \mathrm{~s}^{-1}$ clouds, as well as the CND. The $\mathrm{CH}_{3} \mathrm{OH}$ line complex at $157.2-157.3 \mathrm{GHz}$ has been detected in the +20 and $+50 \mathrm{~km} \mathrm{~s}^{-1}$ clouds but not towards Sgr A*/CND.

Conclusions. A $3 \sigma$ upper limit for the fractional abundance ratio of $\left[\mathrm{O}_{2}\right] /\left[\mathrm{H}_{2}\right]$ is found to be $X\left(\mathrm{O}_{2}\right) \leq 1.2 \times 10^{-7}$ towards the Sgr A molecular belt region.
\end{abstract}

Key words. Galaxy: center - ISM: individual objects: Sgr A - ISM: molecules - ISM: clouds - ISM: abundances

\section{Introduction}

The Odin submillimetre/millimetre wave spectroscopy astronomy and aeronomy satellite has been in operation since 2001. The satellite has been described in detail by Frisk et al. (2003) and the receiver calibration by Olberg et al. (2003). Its prime astronomical priorities were to search the interstellar medium for water vapour $\left(\mathrm{H}_{2} \mathrm{O}\right)$ at a frequency of $556.93600 \mathrm{GHz}$ and molecular oxygen $\left(\mathrm{O}_{2}\right)$ at a frequency of $118.75034 \mathrm{GHz}$, both frequencies not observable from the ground. The $\mathrm{H}_{2} \mathrm{O}$ observations have been abundantly successful, a summary of which has been presented by Hjalmarson et al. (2003, 2007). Emission and absorption in the $557-\mathrm{GHz}{ }_{2}^{16} \mathrm{O}$ line have been detected in the Galactic centre towards the Sgr A* circumnuclear disk (CND), and the Sgr A $+20 \mathrm{~km} \mathrm{~s}^{-1}$ and $+50 \mathrm{~km} \mathrm{~s}^{-1}$ molecular clouds (Sandqvist et al. 2003, 2006). Good reviews on the Galactic centre region have been presented by Morris \& Serabyn (1996) and Mezger et al. (1996). Upper limits have been achieved for the $\mathrm{O}_{2}$ abundances in a number of regions, our first upper limits being of the order of $\leq 10^{-7}$ (Pagani et al. 2003). However, for one source, namely the $\rho$ Oph A molecular cloud, a very weak $\mathrm{O}_{2}$ line has actually been detected (Larsson et al. 2007).

\footnotetext{
* Based on observations with Odin, a Swedish-led satellite project funded jointly by the Swedish National Space Board (SNSB), the Canadian Space Agency (CSA), the National Technology Agency of Finland (Tekes) and Centre National d'Études Spatiales (CNES). The Swedish Space Corporation was the industrial prime contractor and is also responsible for the satellite operation.
}

In this paper, we report the results of Odin observations using the on-board $118-\mathrm{GHz}$ radiometer made towards the Sgr A complex in the Galactic centre.

\section{Observations}

The observations were performed during 26 March-9 April 2003 and 17-26 February 2004 and were part of a larger Galactic centre project The receiver used was the 118-GHz cryo-cooled HEMT receiver with a single-sideband (SSB) temperature of $\approx 730 \mathrm{~K}$ in 2003 and $\approx 840 \mathrm{~K}$ in 2004 . The backend spectrometer for the 118-GHz receiver consisted of an acousto-optical spectrometer (AOS) with a total bandwidth of $1.05 \mathrm{GHz}$ and a channel resolution of $1 \mathrm{MHz}$. At the $118-\mathrm{GHz}$ frequency, the angular resolution of the Odin beam is about $10^{\prime}$ and the main beam efficiency is 0.91 (Frisk et al. 2003).

Three positions were observed, separated by a few arcminutes. The total-power position-switching observing mode was used with a duty cycle of $120 \mathrm{~s}$, the empty reference OFF-position being at $\alpha(\mathrm{B} 1950.0)=17^{\mathrm{h}} 40^{\mathrm{m}} 26^{\mathrm{s}} 8, \delta(\mathrm{B} 1950.0)=$ $-28^{\circ} 35^{\prime} 04^{\prime \prime}$. The 1950.0 coordinates of the three source positions are given in Table 1, and Fig. 1 shows these positions superimposed upon a continuum/molecular distribution map of the Sgr A complex. Most of the features in the Sgr A complex seen in this figure are within the $118-\mathrm{GHz} 10^{\prime}$-beam at all three positions. Due to different problems with the AOS in the 2003 period only 12 orbits (hours) of observation produced useful data (eight towards Sgr A* CND, three towards the $+20 \mathrm{~km} \mathrm{~s}^{-1}$ cloud and 
Table 1. Observed positions in the Galactic centre Sgr A region.

\begin{tabular}{lll}
\hline \hline & $\alpha(\mathrm{B} 1950.0)$ & $\delta(\mathrm{B} 1950.0)$ \\
\hline Sgr A* circumnuclear disk & $17^{\mathrm{h}} 42^{\mathrm{m}} 29^{\mathrm{s}} .3$ & $-28^{\circ} 59^{\prime} 18^{\prime \prime}$ \\
Sgr A $+20 \mathrm{~km} \mathrm{~s}^{-1}$ cloud & $17^{\mathrm{h}} 42^{\mathrm{m}} 29^{\mathrm{s}} .3$ & $-29^{\circ} 02^{\prime} 18^{\prime \prime}$ \\
Sgr A $+50 \mathrm{~km} \mathrm{~s}^{-1}$ cloud & $17^{\mathrm{h}} 42^{\mathrm{m}} 41^{\mathrm{s}} .0$ & $-28^{\circ} 58^{\prime} 00^{\prime \prime}$ \\
\hline
\end{tabular}

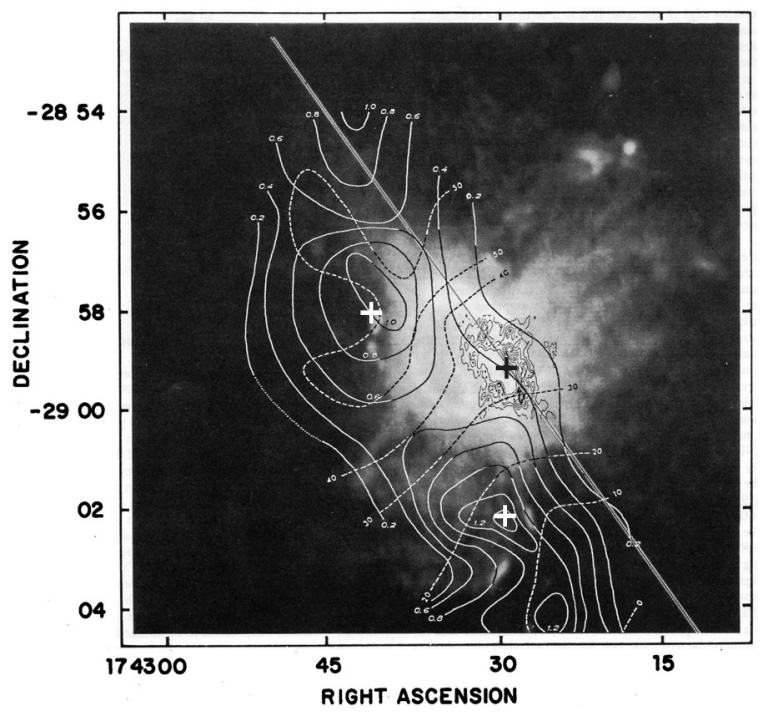

Fig. 1. The three observed positions in the Galactic centre Sgr A region are marked by crosses, the black cross indicating also Sgr A* The Sgr A complex consists of the Sgr A*, Sgr A West, Sgr A East and Sgr A halo radio continuum sources (20-cm continuum radiograph), the circumnuclear disk (CND, $\mathrm{HCN}$ - thin contours), and the molecular belt (2- $\mathrm{mm} \mathrm{H}_{2} \mathrm{CO}$ - thick contours). Isovelocity contours are indicated with dashed contours. The straight diagonal line indicates the orientation of the Galactic plane. The coordinates are epoch 1950.0. (adapted from Sandqvist 1989).

one towards the $+50 \mathrm{~km} \mathrm{~s}^{-1}$ cloud). During the 2004 period a total of 77 orbits produced useful data (divided equally amongst the three above-mentioned positions).

The $118-\mathrm{GHz}$ receiver on board Odin is not properly phaselocked but slowly drifts in frequency. However, during $40 \%$ of Odin's observing time, the satellite is looking at the Earth's atmosphere. This allows us to monitor the stability of the receiver and to correct for any frequency drift using the telluric oxygen line. Larsson et al. $(2003,2007)$ have given a detailed description of this method of frequency calibration.

In February/March 2007, Odin observed the Sgr A $+50 \mathrm{~km} \mathrm{~s}^{-1}$ cloud during 150 orbits with one of the front end submillimetre receivers tuned to $486.79638 \mathrm{GHz}$, the rest frequency of the $\left(3_{3}-1_{2}\right) \mathrm{O}_{2}$ line, using the position-switching method. The back end spectrometer was an autocorrelator (AC2) with a total bandwidth of $700 \mathrm{MHz}$ and a channel resolution of $1.2 \mathrm{MHz}\left(0.62 \mathrm{~km} \mathrm{~s}^{-1}\right)$. The SSB system temperature was about $3000 \mathrm{~K}$. The angular resolution of the Odin beam at this frequency is 2.39 and the main beam efficiency is 0.89 .

Supplementary ground-based observations in the $157-\mathrm{GHz}$ band were performed with 12-m millimetre wave telescope of the Arizona Radio Observatory at Kitt Peak in March 2004 to check a suspicious feature seen in the 2003 Odin $119-\mathrm{GHz}$ profile near the frequency expected for a possible $\mathrm{SiC}$ feature (see Fig. 1.1 of Hjalmarson et al. 2004). The 2-mm receiver (133-180 GHz), with two orthogonal front ends, was tuned to $157.4941010 \mathrm{GHz}$, the expected rest frequency of the ${ }^{3} \Pi_{2}, J=4-3 \mathrm{SiC}$ line (Pickett et al. 1998). It had a nominal

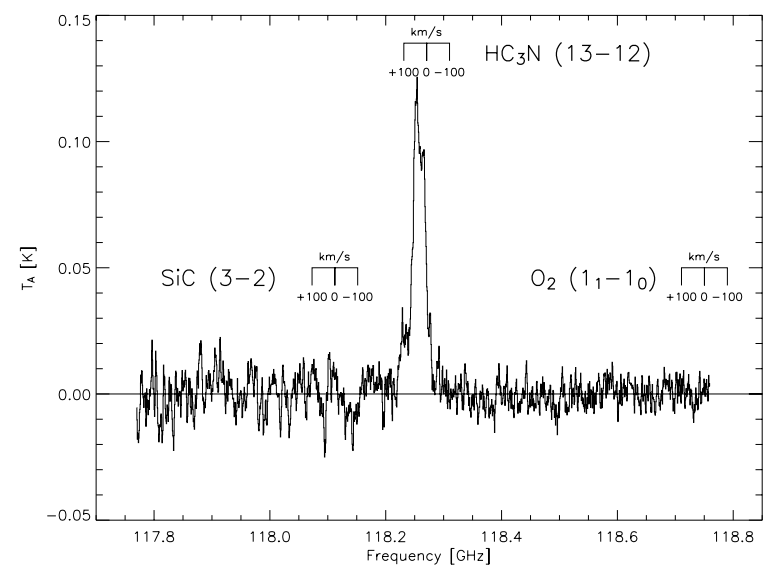

Fig. 2. Odin 118-GHz observations of the Sgr A complex region in the Galactic centre - total integrated profile, channel resolution: $1.6 \mathrm{~km} \mathrm{~s}^{-1}$. Small velocity scales are shown above each expected position of line features.

SSB receiver temperature of about $125 \mathrm{~K}$. The total system temperature varied between 600 and $1500 \mathrm{~K}$, depending on the elevation of the source during the observations. The backend spectrometer was the Millimeter Autocorrelator (MAC) operated in parallel mode with useable bandwidth of $600 \mathrm{MHz}$ and an effective channel resolution of $0.74 \mathrm{~km} \mathrm{~s}^{-1}$. Again the positionswitching observation mode was used. The beamwidth of the $12-\mathrm{m}$ telescope at $157 \mathrm{GHz}$ is $40^{\prime \prime}$.

\section{Results}

The 2004 observations at the three positions were co-added after the careful frequency calibrations outlined in the previous section. The resulting profile is shown in Fig. 2. The intensity scale has not been corrected for the main beam efficiency $(\approx 0.9)$ and a weak second-order polynomial baseline has been subtracted. The AOS has some low-level system instability which is variable in amplitude and frequency over the band. To obtain a signal-free OFF position in the Galactic centre region, a large switching angle $\left(0^{\circ} .6\right)$ had to be used resulting in a switching period of $120 \mathrm{~s}$. Due to this, some of the system instability may not have been completely removed, causing a non-uniform rms noise level across the AOS band.

The strong line present in Fig. 2 we propose is identified as the $J=13-12$ transition of the $\mathrm{HC}_{3} \mathrm{~N}$ (cyanoacetylene) molecule which has a rest frequency of $118.2707322 \mathrm{GHz}$. This line was also very clear in the 2003 data, which has been published in a preliminary report on Odin observations by Hjalmarson et al. (2004). In that profile there appeared also to be a weak broader feature at the lower frequency side of the $\mathrm{HC}_{3} \mathrm{~N}$ line which was suspected to possibly be the ${ }^{3} \Pi_{2}$, $J=3-2$ ground state transition of the $\mathrm{SiC}$ molecule with a rest frequency of $118.1122437 / 93 \mathrm{GHz}$. At the high frequency end of the observed band of that profile there was also a hint of emission from the $\mathrm{O}_{2}$ molecule whose $\left(1_{1}-1_{0}\right)$ transition has a rest frequency of $118.7503430 \mathrm{GHz}$. All quoted rest frequencies are taken from the on-line JPL Molecular Spectroscopy Catalogue at http://spec.jpl.nasa.gov/ (Pickett et al. 1998). In the much more sensitive profile of the 2004 data presented here in Fig. 2, there are no obvious indications of these $\mathrm{SiC}$ and $\mathrm{O}_{2}$ lines (whose expected frequency intervals are marked by the individual velocity scales) and we deem that uncertainties in the baseline caused these suspected features in the 2003 data profile. 

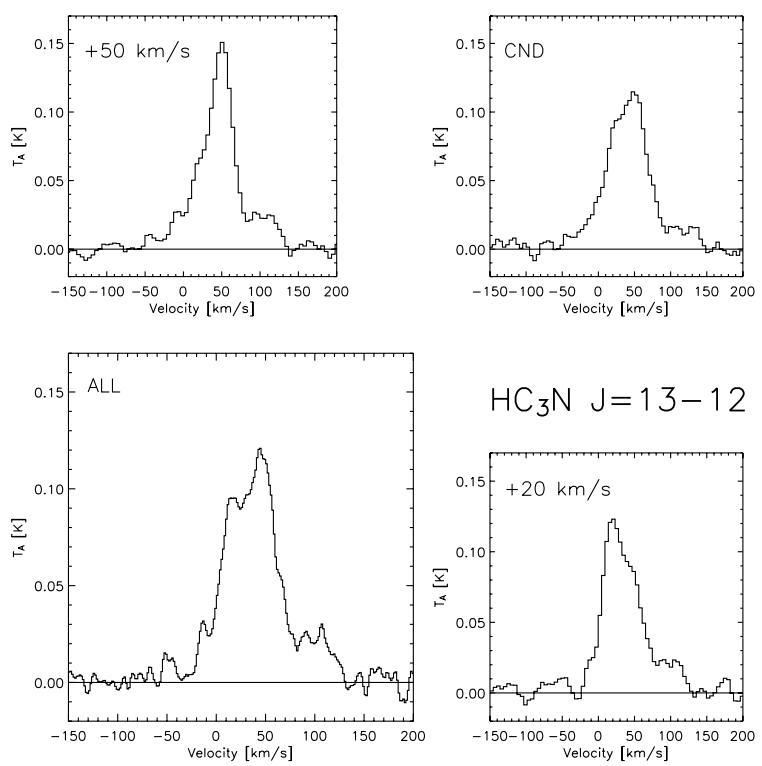

Fig. 3. $\mathrm{HC}_{3} \mathrm{~N}$ "map" showing the three profiles obtained towards the $+50 \mathrm{~km} \mathrm{~s}^{-1}$ cloud (upper left), Sgr A* CND (upper right) and the $+20 \mathrm{~km} \mathrm{~s}^{-1}$ cloud (lower right) - channel resolution is $4.7 \mathrm{~km} \mathrm{~s}^{-1}$. The total integrated profile with a channel resolution of $1.6 \mathrm{~km} \mathrm{~s}^{-1}$ is shown in the lower left.

\section{1. $\mathrm{HC}_{3} \mathrm{~N}$}

The clarity and sharpness of the strong $\mathrm{HC}_{3} \mathrm{~N}$ feature in Fig. 2 shows that the frequency calibration of the $118-\mathrm{GHz}$ receiver can be considered successful. The feature is also strong enough to permit the limited mapping done in connection with the submillimetre observations. The individual $\mathrm{HC}_{3} \mathrm{~N}$ spectra observed towards the $\mathrm{Sgr} \mathrm{A}^{*} \mathrm{CND}$, the +20 and $+50 \mathrm{~km} \mathrm{~s}^{-1}$ clouds, together with the total integrated profile, are shown in Fig. 3.

The Odin $10^{\prime}$-beamwidth covers practically the whole region shown in Fig. 1 and the map spacing is smaller than thirdbeam-spacing. It is clear from both the velocity halfwidths (which are of the order of $50 \mathrm{~km} \mathrm{~s}^{-1}$ ) and the velocities of the peak temperatures that the $\mathrm{HC}_{3} \mathrm{~N}$ profiles show a behaviour consistent with their dominant sources being the +20 and $+50 \mathrm{~km} \mathrm{~s}^{-1}$ clouds in the molecular belt (cf. the $\mathrm{H}_{2} \mathrm{CO}$ profiles in Sandqvist 1989). These are relatively warm $(\approx 100 \mathrm{~K})$, highdensity $\left(\approx 10^{4.5}\right)$ giant molecular clouds with moderate velocity dispersion $\left(\approx 25 \mathrm{~km} \mathrm{~s}^{-1}\right)$ which, however, have only few signs of star formation.

\section{2. $\mathrm{O}_{2}$}

No $\mathrm{O}_{2}$ line was detected down to a $T_{\mathrm{mb}}$ rms limit of $4.8 \mathrm{mK}$, a value determined for the frequency range of 118.400-118.752 GHz. Unfortunately, the slow frequency drift of the Odin 119-GHz receiver had moved the $\mathrm{O}_{2}$ frequency to the very edge of the observing band in 2004 and thus only the positive $\mathrm{O}_{2}$ velocity range (and a small amount of the negative $\mathrm{O}_{2}$ velocity range) was observable. However, molecular emission from the Sgr A complex dominates in the positive velocity range (see e.g. Fig. 3). So, if any $\mathrm{O}_{2}$ were present in the Sgr A complex it would have been included in this profile. At this frequency, the 10 arcmin Odin beam includes all three Sgr A complex components (see Fig. 1).

The 487- $\mathrm{GHz} \mathrm{O}_{2}$ observations, on the other hand, have an angular resolution of only 2.4 and are thus limited to the

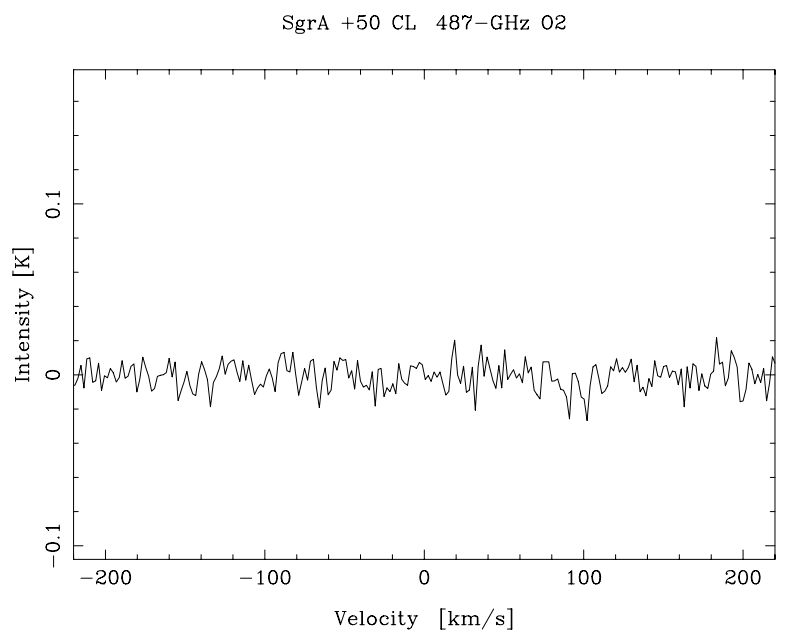

Fig. 4. Odin 487-GHz $\mathrm{O}_{2}$ observations of the $\mathrm{Sgr} \mathrm{A}+50 \mathrm{~km} \mathrm{~s}^{-1}$ cloud. Channel resolution: $1.8 \mathrm{~km} \mathrm{~s}^{-1}$.

component at which Odin is pointing. The profile observed towards the Sgr A $+50 \mathrm{~km} \mathrm{~s}^{-1}$ cloud is presented in Fig. 4 and it is quite featureless. This profile has been boxcar-smoothed and no $\mathrm{O}_{2}$ line was detected down to a $T_{\mathrm{mb}} \mathrm{rms}$ limit of $9.0 \mathrm{mK}$.

\section{3. $\mathrm{SiC}$ and $\mathrm{CH}_{3} \mathrm{OH}$}

It is quite clear from Fig. 2 that there is no obvious detection of 118-GHz ground-state emission from $\mathrm{SiC}$ in the Sgr A complex. Due to the non-uniformity of the rms noise across the Odin 118-GHz band (see above), calculating a $T_{\mathrm{mb}} \mathrm{rms}$ limit for the ground state $\mathrm{SiC}$ line was limited to the frequency range 117.8-118.2 GHz, yielding a value of $8.7 \mathrm{mK}$.

The attempt to observe the higher SiC transition with the ARO 12-m mm-wave telescope was motivated by the noisy 2003 Odin observations as mentioned in Sect. 2 and was carried out before we had access to the 2004 Odin observations. Although no detection of a $\mathrm{SiC}$ line was made, we nonetheless present the results of these ARO observations here. The observations made with the two orthogonal front ends were averaged together. These observations did serendipitously result in the detection of the strong $\mathrm{CH}_{3} \mathrm{OH}$ line complex at $157.2-157.3 \mathrm{GHz}$ in the $+20-$ and $+50-\mathrm{km} \mathrm{s}^{-1}$ clouds but not towards the CND. The lack of any $\mathrm{CH}_{3} \mathrm{OH}$ emission towards the CND position is not really surprising since the $40^{\prime \prime}$ beam of the ARO 12-m telescope would be mostly looking at the cavity inside the CND. The baselines including a dominant standing wave were removed by assuming a featureless profile towards the CND cavity, - based on the lack of any $\mathrm{CH}_{3} \mathrm{OH}$ feature towards the $\mathrm{CND}$ as mentioned above super-smoothing it by Hanning it 19 times, and then subtracting the result from all three profiles. The results are shown in Fig. 5. The $T_{\mathrm{mb}}$ rms limits in the three profiles are 20,11 and $19 \mathrm{mK}$ in the $+50 \mathrm{~km} \mathrm{~s}^{-1}$ cloud, CND and $+20 \mathrm{~km} \mathrm{~s}^{-1}$ cloud, respectively, assuming a main beam efficiency of 0.7 .

\section{Discussion}

\section{1. $\mathrm{HC}_{3} \mathrm{~N}$}

The $\mathrm{HC}_{3} \mathrm{~N}$ molecule has many transitions which can be observed from the ground and it is considered to be an excellent density probe with optically thin lines (Vanden Bout et al. 1983). Walmsley et al. (1986) have made measurements of nine of these transitions towards selected regions in the Sgr A molecular belt, 


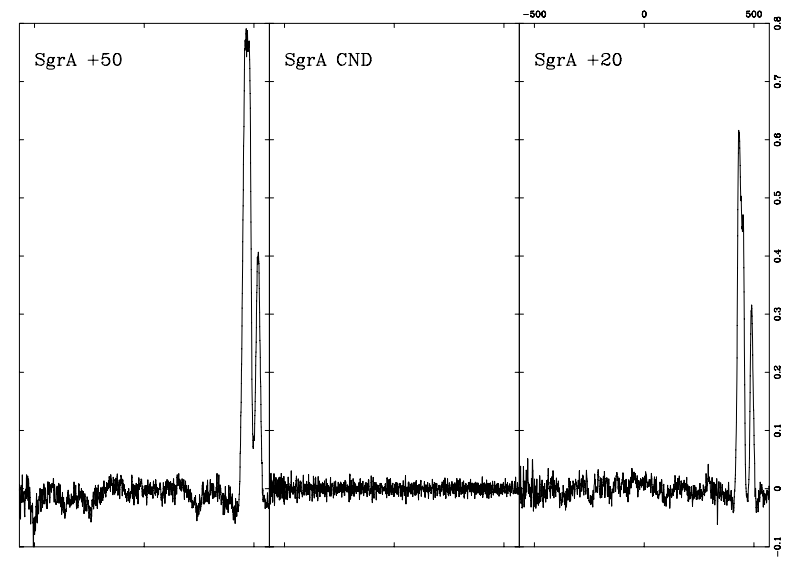

Fig. 5. ARO 12-m telescope $157-\mathrm{GHz}$ observations of the Sgr A complex region in the Galactic centre - towards the $+50 \mathrm{~km} \mathrm{~s}^{-1}$ cloud, the CND and the $+20 \mathrm{~km} \mathrm{~s}^{-1}$ cloud. The velocity $x$-axis ranges from -550 to $+550 \mathrm{~km} \mathrm{~s}^{-1}$ assuming the $\mathrm{SiC}$ rest frequency as the reference and the intensity $y$-axis ranges from -0.1 to $+0.8 \mathrm{~K}$. Channel resolution: $0.74 \mathrm{~km} \mathrm{~s}^{-1}$. The strong lines near $+500 \mathrm{~km} \mathrm{~s}^{-1}$ originate from the $\mathrm{CH}_{3} \mathrm{OH}$ line complex at $157.2-157.3 \mathrm{GHz}$.

ranging in frequency from 9 to $226 \mathrm{GHz}$. From a statistical equilibrium model they derived a density of $10^{4} \mathrm{~cm}^{-3}$ for the +20 and $+50 \mathrm{~km} \mathrm{~s}^{-1}$ clouds assuming a temperature of $80 \mathrm{~K}$. The fit could be slightly improved non-uniquely by assuming that $20 \%$ of the mass is in clumps with a density of $10^{5} \mathrm{~cm}^{-3}$ with the remaining $80 \%$ at a density of $5 \times 10^{3} \mathrm{~cm}^{-3}$.

Two of their transitions, (12-11) and (15-14), stradle our (13-12) transition but they were observed with the Texas 4.9-m Millimetre Wave Observatory (MWO) telescope which has five times better resolution $(\approx 2 \mathrm{arcmin})$ than Odin. The Odin profiles towards the +20 and $+50 \mathrm{~km} \mathrm{~s}^{-1}$ clouds have intensity maxima at radial velocities of +20 and $+50 \mathrm{~km} \mathrm{~s}^{-1}$, respectively. The integrated line intensity of the Odin $J=13-12 \mathrm{HC}_{3} \mathrm{~N}$ total profile (ALL in Fig. 3) was found to be about $8.8 \mathrm{~K} \mathrm{~km} \mathrm{~s}^{-1}$ assuming a main beam efficiency of 0.91 . The elongated shape and angular dimensions of the molecular belt are well established from different molecular observations (e.g. Fig. 1). We will here approximate the size with a spherical, homogeneous cloud of diameter of 6.4 arcmin or $16 \mathrm{pc}$, assuming a distance of $8.5 \mathrm{kpc}$ to the Galactic centre. This corresponds to an Odin beam dilution of 0.25 . Correcting for this beam dilution we match the modelled $J=13-12$ intensity of about $35 \mathrm{~K} \mathrm{~km} \mathrm{~s}^{-1}$ of Walmsley et al. (1986). The Walmsley et al. best-fit model we adopt here is their single-component model having the parameters: gas temperature $T=80 \mathrm{~K}$, uniform gas density $n_{\mathrm{H} 2}=10^{4} \mathrm{~cm}^{-3}$, and a fractional $\mathrm{HC}_{3} \mathrm{~N}$ abundance per velocity gradient of $10^{-9}\left(\mathrm{~km} \mathrm{~s}^{-1} \mathrm{pc}^{-1}\right)^{-1}$. We find then a molecular hydrogen column density through the centre of the model cloud $N\left(\mathrm{H}_{2}\right)$ of $5 \times 10^{23} \mathrm{~cm}^{-2}$, and since the whole model cloud is encompassed by our beam we observe a total mass of $10^{6} M_{\odot}$. Lis \& Carlstrom (1994) find $N\left(\mathrm{H}_{2}\right)$ values of $4.1 \times 10^{23} \mathrm{~cm}^{-2}$ for the $+20 \mathrm{~km} \mathrm{~s}^{-1}$ cloud and $2.4 \times$ $10^{23} \mathrm{~cm}^{-2}$ for the $+50 \mathrm{~km} \mathrm{~s}^{-1}$ cloud from their observations of the $800-\mu \mathrm{m}$ continuum emission and they deduce masses of $5 \times$ $10^{5} M_{\odot}$ and $4 \times 10^{5} M_{\odot}$, respectively, for these two components of the molecular belt. Finally, by using a velocity gradient of $2 \mathrm{~km} \mathrm{~s}^{-1} \mathrm{pc}^{-1}$ (cf. the isovelocity contours of Fig. 1) we estimate the $\mathrm{HC}_{3} \mathrm{~N}$ fractional abundance to be $2 \times 10^{-9}$. Hence the central $\mathrm{HC}_{3} \mathrm{~N}$ column density for the molecular belt model source is $N\left(\mathrm{HC}_{3} \mathrm{~N}\right)=10^{15} \mathrm{~cm}^{-2}$.

\section{2. $\mathrm{O}_{2}$}

In order to make an estimate of the upper limit of the $\mathrm{O}_{2}$ abundance, implied by our non-detections of the $119-$ and $487-\mathrm{GHz}$ lines, we use as the starting point the $\mathrm{HC}_{3} \mathrm{~N}$ modelling results of Walmsley et al. (1986) and our results for the $\mathrm{HC}_{3} \mathrm{~N} J=$ 13-12 line as presented in the previous section. We then adjust the $\mathrm{O}_{2}$ abundance in our model cloud until we obtain a predicted maximum intensity for the $119-\mathrm{GHz} \mathrm{O}_{2}$ line, as observed with the Odin $10^{\prime}$ beam, which is compatible with the rms noise level of our observation. Our $4.8 \mathrm{mK}$ rms noise value of the nondetected $119-\mathrm{GHz} \mathrm{O}_{2}$ observation then yields a $3 \sigma$ upper limit for the $\mathrm{O}_{2}$ fractional abundance of $X\left(\mathrm{O}_{2}\right) \leq 1.2 \times 10^{-7}$. The $3 \sigma$ upper limit for the central $\mathrm{O}_{2}$ column density in the model cloud is $N\left(\mathrm{O}_{2}\right) \leq 6 \times 10^{16} \mathrm{~cm}^{-2}$. This model also results in a predicted $487-\mathrm{GHz} \mathrm{O}_{2}$ line main beam temperature of $T_{\mathrm{mb}} \approx 1 \mathrm{mK}$, well below the rms noise of $9 \mathrm{mK}$ in our $487-\mathrm{GHz}$ observation.

The first Odin $\mathrm{O}_{2}$ non-detection results improved the earlier SWAS upper limits in star forming regions by a factor of a few and in cold dark clouds by factors of between 20 and 40 (Pagani et al. 2003; Goldsmith et al. 2000). The Odin results gave $\mathrm{O}_{2}$ abundance upper limits of $X\left(\mathrm{O}_{2}\right)$ between $5 \times 10^{-8}$ and $8 \times 10^{-7}$ for different Galactic sources. In the Small Magellanic Cloud, Wilson et al. (2005) found an upper limit of the $\mathrm{O}_{2}$ abundance ratio of $1.3 \times 10^{-6}$. The only source in which $\mathrm{O}_{2}$ has actually been detected, through its $119-\mathrm{GHz}$ transition, is $\rho$ Oph A, where Odin found an $\mathrm{O}_{2}$ abundance ratio of $X\left(\mathrm{O}_{2}\right)=5 \times 10^{-8}$ (Larsson et al. 2007).

The Sgr A complex contains a variety of conditions potentially favourable for relatively high $\mathrm{O}_{2}$ abundance. One such region is the existence of the shock front interaction between the expansion of the Sgr A East shell into the molecular belt and the centrally peaked thermal X-ray emission from the interior of the Sgr A East shell (Maeda et al. 2002). The $\mathrm{O}_{2}$ could be a tracer of the X-rays in the warm envelope of the molecular belt facing Sgr A East (see e.g. Goldsmith et al. 2002 for a discussion on shock-produced $\mathrm{O}_{2}$ or Stäuber et al. 2005 for a discussion on $\mathrm{X}$-ray-induced chemical models). On the other hand, the UV radiation field emitted by the central cluster of hot massive stars (the IRS16 stars) (Allen et al. 1990) strikes the surface of the molecular belt with the result that the surface of the $+50 \mathrm{~km} \mathrm{~s}^{-1}$ cloud which faces Sgr A* and IRS16 is bright in [CII] line emission - as if this part of the cloud is directly photo-dissociated by the UV radiation from the Centre itself (Genzel et al. 1990). This would have a negative effect on the production of $\mathrm{O}_{2}$ in this part of the molecular belt's envelope. But all this would only be valid for the relatively thin inner envelope of the molecular belt, much beyond the resolution of the Odin beam.

Consider now the whole molecular belt. Unlike many other giant molecular clouds in the Galaxy, the molecular belt cloud components do not contain any significant embedded massive star formation regions which could induce increased $\mathrm{O}_{2}$ abundance by heating surrounding dust complexes. In fact, it has been shown in a number of observations that, again differing from many other Galactic regions, the Sgr A complex molecular clouds have considerably lower dust temperatures than gas temperatures (e.g. Güsten et al. 1985). So, instead of $80 \mathrm{~K}$, which is the gas temperature that we use in our abundance determination above, the actual dust temperature is more like $30 \mathrm{~K}$ (Lis \& Carlstrom 1994).

Hollenbach et al. (2008) have studied the oxygen chemistry in clouds irradiated by an external FUV flux. This model includes photodissociation, standard gas phase chemistry, freezeout of species on grain surfaces, some limited grain surface 
chemistry and some desorption mechanisms. Hollenbach et al. have kindly applied their model to our case. In their standard case for a cloud with density of $10^{4} \mathrm{~cm}^{-3}$ and an external FUV field which is 100 times greater than the local ISRF, they obtain at the surface a gas temperature of $120 \mathrm{~K}$ and a dust temperature of $31 \mathrm{~K}$, but at the place where the $\mathrm{H}_{2} \mathrm{O}$ and the $\mathrm{O}_{2}$ abundances peak they get a gas temperature of $22 \mathrm{~K}$ and a dust temperature of $15 \mathrm{~K}$. Increasing the external radiation field until they get a dust temperature of $30 \mathrm{~K}$ at the $\mathrm{O}_{2}$ peak, they find that the column density of $\mathrm{O}_{2}$ in the cloud has a value of $N\left(\mathrm{O}_{2}\right)=2 \times 10^{16} \mathrm{~cm}^{-2}$. This is a factor of three lower than our upper limit. So, it is not unreasonable that we were unable to detect the $\mathrm{O}_{2}$ line, assuming the validity of these models.

\section{3. $\mathrm{SiC}$}

It was not until 1987 that the $\mathrm{SiC}$ molecule was spectroscopically identified in the laboratory (Bernath et al. 1988). The $\mathrm{SiC}$ molecule has not previously been observed in the interstellar medium. Only in the circumstellar envelope of the carbon star IRC +10216 and the evolved carbon star CIT- 6 has SiC been detected, and then not at the ground-level frequency (Cernicharo et al. 1989). In a manner similar to the $\mathrm{SiO}$ molecule, it may be that strong shocks disrupting dust grains are required to release $\mathrm{SiC}$ into the gaseous interstellar medium. Martin-Pintado et al. (1997) have observed that $\mathrm{SiO}$ emission in the Galactic centre regions seems to delineate closely the nonthermal Radio Arc and also there is a region of enhanced $\mathrm{SiO}$ emission close to $\mathrm{Sgr} \mathrm{A}^{*}$. There is nonetheless also $\mathrm{SiO}$ emission coming from the +20 and $+50 \mathrm{~km} \mathrm{~s}^{-1}$ clouds. It is not unreasonable to suspect that $\mathrm{SiC}$ may coexist in the $\mathrm{SiO}$ regions.

The upper limits implied by the non-detection of $\mathrm{SiC}$ at $118 \mathrm{GHz}$ and $157 \mathrm{GHz}$ were determined from the spectroscopic data of the Cologne Database for Molecular Spectroscopy (Müller et al. 2005). The line intensities are based on an ab initio dipole moment of 1.7 D from an early calculation (Cernicharo et al. 1989). Note that both the Cologne and JPL databases are essentially identical for $\mathrm{SiC}$. A more recent ab initio calculation gives a very similar value of $1.65 \mathrm{D}$ for the equilibrium dipole moment (Pramanik \& Das 2007), so the Einstein A values given by the Cologne database were used $\left(A_{3-2}=1.268 \times 10^{-5} \mathrm{~s}^{-1}\right.$ and $A_{4-3}=4.385 \times 10^{-5} \mathrm{~s}^{-1}$ ).

Assuming an excitation temperature of $80 \mathrm{~K}$ and the corresponding partition function of 280 gives upper limit column densities of $16 \times 10^{13}$ molecules $\mathrm{cm}^{-2}$ for the ${ }^{3} \Pi_{2}, J=3-2$, $118 \mathrm{GHz}$ line ( $25 \%$ beam dilution) and $4 \times 10^{13}$ molecules $\mathrm{cm}^{-2}$ for $J=4-3$ at $157 \mathrm{GHz}$ (no beam dilution). These values are based on limiting rms noise levels of $8.7 \mathrm{mK}$ and $20 \mathrm{mK}$ over $100 \mathrm{~km} \mathrm{~s}^{-1}$ for Odin and ARO, respectively, and taking the two unresolved $\Lambda$-doublets of equal intensity into account. These upper limits compare favourably with the observed column density of $6 \times 10^{13}$ molecules $\mathrm{cm}^{-2}$ in IRC+10216 (Cernicharo et al. 1989). A $3 \sigma$ upper limit for the fractional abundance ratio of $[\mathrm{SiC}] /\left[\mathrm{H}_{2}\right]$ in the Sgr A molecular belt is then estimated to be $X(\mathrm{SiC}) \leq 2 \times 10^{-10}$, using the $\mathrm{H}_{2}$ column density derived in Sect. 4.1.

From our upper limit to the $\mathrm{SiC}$ column density of $4 \times$ $10^{13} \mathrm{~cm}^{-2}$ and the $\mathrm{SiO}$ column density range $(7-30) \times 10^{13} \mathrm{~cm}^{-2}$ determined by Martin-Pintado et al. (1997) we arrive at an upper limit to the $\mathrm{SiC} / \mathrm{SiO}$ abundance ratio $(0.13-0.57)$ which is just similar to the standard $\mathrm{C} / \mathrm{O}$ elemental abundance ratio $(0.5)$ listed by Grevesse et al. (1996). Hence deeper SiC searches would be desirable.

\section{Conclusions}

Molecular oxygen remains as elusive as ever, the Galactic centre appears to be no exception. Despite long integrations using the Odin satellite, the $118.75-\mathrm{GHz}$ ground state $\mathrm{O}_{2}$ line has not been detected towards the Sgr A complex, nor has the 487-GHz $\mathrm{O}_{2}$ line. We find a $3 \sigma$ upper limit for the $\mathrm{O}_{2}$ column density in the Sgr A molecular belt of $N\left(\mathrm{O}_{2}\right) \leq 6 \times 10^{16} \mathrm{~cm}^{-2}$, which is compatible with the results of new theoretical oxygen chemistry models. The $3 \sigma$ upper limit for the fractional abundance ratio of $\left[\mathrm{O}_{2}\right] /\left[\mathrm{H}_{2}\right]$ is $X\left(\mathrm{O}_{2}\right) \leq 1.2 \times 10^{-7}$.

Acknowledgements. We should like to express our gratitude to D. Hollenbach et al. for having applied their model to the Sgr A complex molecular belt and for having made available results to us before publication. We also thank the referee for constructive comments. The Kitt Peak 12-m millimetre wave telescope is operated by the Arizona Radio Observatory (ARO), Steward Observatory, University of Arizona.

\section{References}

Allen, D. A., Hyland, A. R., \& Hillier, D. J. 1990, MNRAS, 244, 706 Bernath, P. F., Rogers, S. A., O’Brien, L. C., Brazier, C. R., \& McLean, A. D. 1988, Phys. Rev. Lett., 60, 197

Cernicharo, J., Gottleib, C. A., Guelin, M., Thaddeus, P., \& Vrtilek, J. M. 1989, ApJ, 341, L25

Frisk, U., Hagström, M., Ala-Laurinaho, J., et al. 2003, A\&A, 402, L27

Genzel, R., Stacey, G. J., Harris, A. I., et al. 1990, ApJ, 356, 160

Goldsmith, P. F., Melnick, G. J., Bergin, E. A., et al. 2000, ApJ, 539, L123

Goldsmith, P. F., Li, D., Bergin, E. A., et al. 2002, ApJ, 576, 814

Grevesse, N., Noels, A., \& Sauval, A. J. 1996, in Cosmic Abundances, ed. S. S. Holt, \& G. Sonneborn, ASP Conf. Ser., 99, 117

Güsten, R., Walmsley, C. M., Ungerechts, H., \& Churchwell, E. 1985, A\&A, 143,381

Hjalmarson, Å., Frisk, U., Olberg, M., et al. 2003, A\&A, 402, L39

Hjalmarson, A., Olberg, M., \& the Odin Team 2004, in The Dense Interstellar Medium in Galaxies, the 4th Cologne-Bonn-Zermatt Symposium 2003, ed. S. Pfalzner, C. Kramer, C. Staubmeier, \& A. Heithausen, Springer Proc. in Phys., 91, 331

Hjalmarson, Å., Olberg, M., Florén, H.-G., et al. 2007, Adv. Space Res., 40, 630 Hollenbach, D., Kaufman, M., Bergin, E., \& Melnick, G. 2008, ApJ, submitted Larsson, B., Liseau, R., Bergman, P. et al. 2003, A\&A, 402, L69 Larsson, B., Liseau, R., Pagani, L., et al. 2007, A\&A, 466, 999 Lis, D. C., \& Carlstrom, J. E. 1994, ApJ, 424, 189

Maeda, Y., Baganoff, F. K., Feigelson, E. D., et al. 2002, ApJ, 570, 671

Martin-Pintado, J., de Vicente, P., Fuente, A., \& Planesas, P. 1997, ApJ, 482, L45

Mezger, P. G., Duschl, W. J., \& Zylka, R. 1996, A\&AR, 4, 289

Morris, M., \& Serabyn, E. 1996, ARA\&A, 34, 645

Müller, H. S. P., Schlöder, F., Stutzki, J., \& Winnewisser, G. 2005, J. Mol. Structure, 742, 215

Olberg, M., Frisk, U., Lecacheux, A., et al. 2003, A\&A, 402, L35

Pagani, L., Olofsson, A. O. H., Bergman, P., et al. 2003, A\&A, 402, L77

Pickett, H. M., Poynter, R. L., Cohen, E. A., et al. 1998, J. Quant. Spectrosc. Rad. Transf., 60, 883

Pramanik, A., \& Das, K. K. 2007, J. Mol. Spectr., 244, 13

Sandqvist, Aa. 1989, A\&A, 223, 293

Sandqvist, Aa., Bergman, P., Black, J., et al. 2003, A\&A, 402, L63

Sandqvist, Aa., Bergman, P., Bernath, P., et al. 2006, in From the Center of the Milky Way to Nearby Low-Luminosity Galactic Nuclei, ed. R. Schödel et al., J. Phys. Conf. Ser., 54, 72

Stäuber, P., Doty, S. D., van Dishoeck, E. F., \& Benz, A. O. 2005, A\&A, 440, 949

Vanden Bout, P., Loren, R. B., Snell, R. L., \& Wootten, A. 1983, ApJ, 271, 161 Walmsley, C. M., Güsten, R., Angerhofer, P., Churchwell, E., \& Mundy, L. 1986, A\&A, 155, 129

Wilson, C. D., Olofsson, A. O. H., Pagani, L., et al. 2005, A\&A, 433, L5 